\title{
Eficiência energética: sistemas de iluminação com LEDs, distribuídos em corrente contínua e utilizando energia fotovoltaica
}

\author{
Energy efficiency: LED lighting systems distributed in \\ direct current and using photovoltaic energy
}

\section{Carolina Lannes Boquimpani \\ Ana Lúcia Torres Seroa da Motta \\ Osvaldo Luiz de Carvalho Souza \\ Carlos Henriques Ventura do Rosário Oliveira}

\section{Resumo}

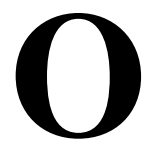

cenário atual é marcado por uma crescente necessidade de energia, esgotamento de recursos naturais e pela necessidade de se aumentar os cuidados com o consumo de energia do setor elétrico. Com o crescimento da demanda por energia, torna-se essencial a obtenção de maior eficiência energética. Para tal é fundamental não somente buscar soluções que produzam energia através de fontes limpas e renováveis, como também mitigar as perdas no sistema elétrico atual. Assim sendo, surge uma proposta de investigar a possibilidade de reestruturar os sistemas de iluminação, utilizando lâmpadas de LED, distribuídos em corrente contínua e abastecidos a partir de energia solar fotovoltaica. O presente artigo se insere nessa linha e apresenta a eficiência gerada pelos sistemas de iluminação constituídos por meio de lâmpadas de LED sem drivers de conversão e energia gerada através de painéis fotovoltaicos. A pesquisa mediu em laboratório as perdas e os ganhos em eficiência dessas lâmpadas de LED. Os resultados experimentais demonstraram ganho na eficiência energética das lâmpadas de LED sem o driver de conversão frente às lâmpadas com drivers. Com o dimensionamento do sistema solar fotovoltaico necessário para o sistema de iluminação, obteve-se o tempo de retorno do investimento.

Palavras-chave: Engenharia civil. Eficiência energética. Lâmpadas de LED. Corrente contínua. Energeia Photovoltaic.

${ }^{1}$ Carolina Lannes Boquimpani ${ }^{1}$ Universidade Federal Fluminense Niteroi - RJ - Brasil https:// orcid. org/ 0000-0002-3254-7161

${ }^{2}$ Ana Lúcia Torres Seroa da Motta ¿Universidade Federal Fluminense Niteroi - RJ - Brasil

${ }^{3}$ Osvaldo Luiz de Carvalho Souza ${ }^{3}$ Universidade Federal Fluminense Niteroi - RJ - Brasil

${ }^{4}$ Carlos Henriques Ventura do Rosário Oliveira 4Universidade Federal Fluminense Niteroi - RJ - Brasil

Recebido em 12/11/18 Aceito em 08/02/19

\section{Abstract}

The current scenario is marked by an increasing need for energy, the depletion of natural resources and the need to increase the attention to energy consumption in the electricity sector. As the the demand for energy increases, it becomes essential to obtain greater energy efficiency. In order to to that, it is fundamental not only to find solutions that produce energy from clean and renewable sources, but also to mitigate losses in the current electrical system. Hence, the idea arises to investigate the possibility of restructuring lighting systems, using LED bulbs distributed in direct current and supplied with solar energy. This article is inserted in this context and presents the efficiency generated by lighting systems consisting of LED bulbs without conversion drivers and energy generated by photovoltaic panels. This research project measured these LED bulbs' losses and gains in efficiency, in laboratory situation. Experimental results demonstrated a gain in the energy efficiency of LED bulbs without the conversion drivers when compared with bulbs with drivers. By sizing the photovoltaic solar system necessary for the lighting system, the investment return time was obtained.

Keywords: Civil engineering. Energy efficiency. LED lamps. Direct current. Photovoltaic Energy. 


\section{Introdução}

Durante o tempo em que o homem explorou os recursos naturais do planeta, o fez sem quaisquer pensamentos no futuro, ocasionando assim inúmeros problemas para o meio ambiente e consequentemente para a população em que nele vive.

A emissão de gases tóxicos, que vem da queima de combustíveis derivados de fontes não renováveis de energia, vem provocando ao longo do tempo o aquecimento global, que afeta o clima e o ecossistema do planeta, trazendo consequências catastróficas ao longo de anos (IMHOFF, 2007).

A energia elétrica é um bem primordial para a sociedade, pois trouxe com ela transformações importantes e permanentes. É, portanto, um bem de grande importância para o desenvolvimento social e econômico de um país (MASARO, 2016).

O consumo de energia de uma sociedade é diretamente proporcional ao seu desenvolvimento econômico e social. Assim, quando uma sociedade tem um ganho na qualidade de vida, como, por exemplo, casas maiores, mais aparelhos eletrônicos e eletrodomésticos, tem-se maior procura por energia e, consequentemente, por eletricidade (OLIVEIRA, 2016).

Um dos setores de maior impacto no balanço energético mundial é o dos centros urbanos, visto que o processo de concentração das cidades e o aumento no consumo de energia os torna responsáveis por $70 \%$ do consumo total e energia (GAVIRIA; PEREIRA; MIZGIER, 2013).

Segundo a EIA (Energy Information Administration), órgão dos Estados Unidos responsável pela coleta, análise e divulgação de informações energéticas, para promover políticas sólidas, mercados eficientes e compreensão pública da energia e sua interação com a economia e o meio ambiente, o consumo de energia elétrica mundial crescerá 28\% entre os anos de 2015 (575 quadrilhões de BTUs) e 2040 (736 quadrilhões de BTUs), conforme apresentado na Figura 1.

Espera-se, segundo a EIA (U.S. ENERGY..., 2017), que a maior parte do aumento na demanda de energia provenha de países não pertencentes à Organization for Economic Co-operation and Development (OCDE), onde o forte crescimento econômico, o aumento do acesso à energia comercializada e as populações em rápido crescimento levam ao aumento da demanda de energia. O consumo de energia em países não pertencentes à OCDE aumentará 41\% entre 2015 e 2040, em contraste com um aumento de 9\% entre 2015 e 2040 nos países da OCDE.

No Brasil, segundo a Empresa de Pesquisa Energética (EMPRESA..., 2015) do Ministério de Minas e Energia (MME), espera-se um crescimento da ordem de 50,6\%, entre os anos de 2015 e 2024 , não obstante a crise econômica instalada nos últimos anos no país. A Tabela 1 mostra a projeção de crescimento do consumo total de eletricidade (incluindo autoprodução) no Brasil considerando os anos de 2015, 2019 e 2024. Observa-se nessa tabela um prognóstico de aumento de $51 \%$ no consumo total de eletricidade no país em apenas nove anos.

Com a expectativa desse crescimento da demanda de energia elétrica, vêm à tona questões como: a maneira como a matriz energética é tratada, o modo como são explorados os recursos naturais que geram energia, a qualidade e a disponibilidade quantitativa dessa energia (MOREIRA; GIOMETTI, 2008).

Figura 1 - Consumo mundial de energia entre 1990 e 2040

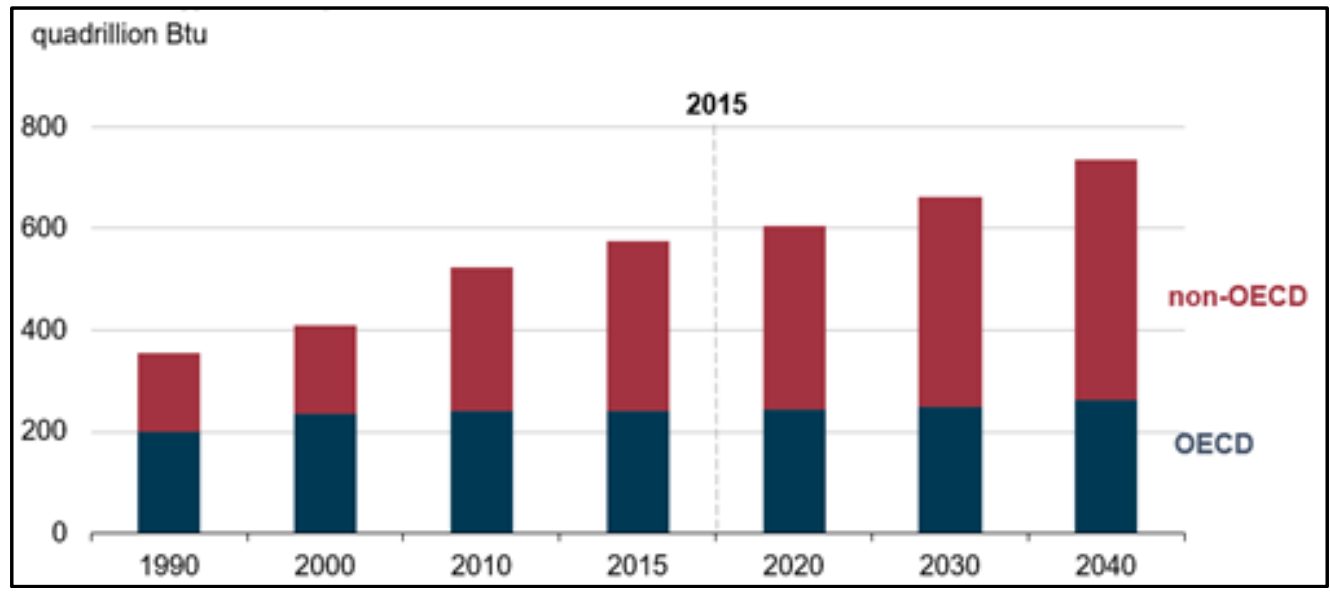

Fonte: EIA - International Energy Outlook (U.S. ENERGY..., 2017). 
Tabela 1 - Projeção do consumo de energia elétrica no Brasil

\begin{tabular}{c|c|c}
\hline Ano & Consumo (TWh) & Prognóstico (\%) \\
\hline 2015 & 524,6 & 100 \\
2019 & 613,4 & 117 \\
2024 & 790,4 & 151 \\
\hline
\end{tabular}

Fonte: adaptado de Empresa de Pesquisa Energética (2015).

Em novos edifícios, a incorporação de tecnologias eficientes desde a concepção inicial do projeto pode resultar em uma economia superior a $50 \%$, quando se compara com edifícios projetados sem o uso dessas tecnologias (LENOIR; THELLIER; GARDE, 2011).

Torna-se interessante buscar soluções para que as unidades consumidoras de energia produzam sua própria demanda de forma mais eficiente e com menores perdas. Portanto, as técnicas que possibilitam melhorias na utilização e também na eficiência da energia elétrica devem ser analisadas e implantadas de acordo com sua viabilidade (ALVES, 2018).

Dispositivos que empregam corrente contínua (CC) nas aplicações finais, tais como os sistemas de iluminação LED (Light Emitting Diode), precisam se adaptar ao atual sistema de distribuição de energia elétrica, o qual se faz em corrente alternada (CA). Para tal, eles possuem acoplados conversores, chamados de drivers. Esses conversores são capazes de transformar a CA, vinda da rede de distribuição-padrão, em CC. Essas conversões ocasionam perdas de eficiência nos dispositivos LED (MONTEIRO; CARVALHO; NOGUEIRA, 2014; PINTO, 2008; NOGUEIRA, 2012).

A utilização de lâmpadas de LED em sistemas de iluminação proporciona redução no consumo de energia, visto que os LEDs são capazes de iluminar de forma mais eficiente e duradoura (MOURA, 2015).

Aliados à característica supracitada das lâmpadas de LED, cabe mencionar que os sistemas solares fotovoltaicos operam em CC, possibilitando, consequentemente, que lâmpadas de LED sejam conectadas diretamente aos painéis solares, prescindindo dos conversores de transformação (CA-CC-CA), o que pode tornar esses sistemas de iluminação mais sustentáveis e eficientes (BOEKE; WENDT; YSEBOOD, 2013).

O objetivo deste artigo é realizar um experimento em laboratório com o propósito de avaliar a eficiência das lâmpadas de LED com e sem o driver de conversão de CA para CC; e comparar a eficiência energética e financeira, calculando o tempo de retorno do investimento, entre um sistema de iluminação em CA, com lâmpadas de LED com drivers de conversão e energia provinda de concessionária com um sistema de iluminação em CC, com lâmpadas de LED sem driver de conversão e energia provinda de um sistema fotovoltaico.

\section{Método}

A metodologia da pesquisa baseou-se na avaliação da eficiência de lâmpadas LEDs, com e sem driver de conversão, e posterior dimensionamento de um sistema solar fotovoltaico necessário à iluminação de um ambiente residencial.

\section{Ensaio em laboratório}

Inicialmente, com o objetivo de avaliar a eficiência das lâmpadas de LED com e sem drivers de conversão, conduziu-se um experimento em laboratório. Os ensaios foram feitos no Laboratório de Luminotécnica (LabLux) da UFF, Universidade Federal Fluminense, o qual possui convênio com a Programa Nacional de Conservação de Energia Elétrica (Procel) e a Eletrobrás, e tem o objetivo de realizar ensaios em produtos na área de luminotécnica para a certificação de eficiência energética.

As três lâmpadas selecionadas para o estudo apresentam diferentes características, a saber: potências, temperaturas de cor e fabricantes:

(a) Lâmpada n. 1: Lâmpada de LED tubular (OUR LUX), SuperLed Tube 20W, 110-240 V, $50 / 60 \mathrm{~Hz}$, Branca neutra $4.000 \mathrm{~K} \mathrm{Fp}>0,92$, fabricação: 08/2016 Lote 025691;

(b) Lâmpada n. 2: Lâmpada de LED tubular (Luminatti by Danuri), 10 W, 127-220 V, $60 \mathrm{~Hz}$, 4000K Fp 0,92, fabricação: 06/2017 Lote 191; e

(c) Lâmpada n. 3: Lâmpada de LED tubular (OUR LUX), SuperLed Tube 10W, 110-240 V, 50/60 Hz, Branca fria 6.500 K Fp > 0,92, fabricação: 12/2017 Lote 035710.

A Figura 2 ilustra as lâmpadas utilizadas nos experimentos.

Nos testes laboratoriais, com a utilização de um multímetro (marca: Eda, modelo: DT9205A), avaliou-se a tensão na qual o LED de cada lâmpada operava. Os valores obtidos nessas medições encontram-se na Tabela 2. 
Figura 2 - Lâmpadas n. 1, n. 2 e n. 3 utilizadas no experimento

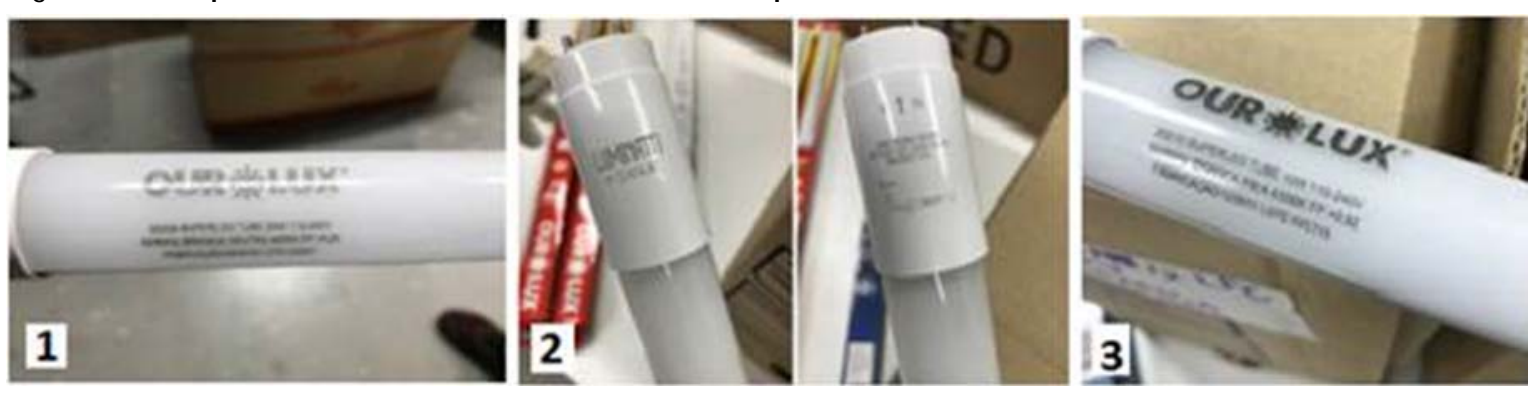

Tabela 2 - Tensão de operação do LED das lâmpadas n. 1, n. 2 e n. 3

\begin{tabular}{c|c}
\hline $\mathbf{N}^{0}$ da lâmpada & Tensão do LED (Volts) \\
\hline 1 & 72,3 \\
2 & 73,2 \\
3 & 56,8 \\
\hline
\end{tabular}

Esses valores de tensões foram, posteriormente, os valores estabelecidos na fonte de corrente contínua para avaliação da eficiência das lâmpadas sem o driver de conversão.

Após a medição da tensão de operação do LED, as lâmpadas, ainda com o driver de conversão, foram individualmente colocadas dentro de uma esfera integrada de Ulbricht (marca: Chuanghui Instrument, modelo: 2m). Esse aparelho é o instrumento óptico que possibilita medir o fluxo luminoso (lm) e calibrar dispositivos que respondam ao espectro luminoso, visível ou não.

A Figura 3 ilustra a lâmpada n. 1 na esfera integrada de Ulbricht.

Objetivando medir os parâmetros elétricos (potência efetiva e corrente), parâmetros radiométricos e fotométricos (fluxo luminoso e eficiência) e colorimétricos (CCT - correlated colour temperature ou temperatura de cor - e IRC - índice de reprodução de cor) das lâmpadas selecionadas, utilizou-se um espectro radiômetro da marca Everfine, modelo Hass-2000, de um wattímetro da marca Yokogawa, modelo WT-210, e de uma fonte em corrente alternada (127 V) da marca Pacific, modelo 110 ADX.

Durante os testes, para que o fluxo de energia se estabilizasse nos equipamentos de medição, foi necessário aguardar aproximadamente quinze minutos a fim de obter dados precisos. A não observância desse intervalo de tempo acarretaria a imprecisão dos dados obtidos, em consequência da oscilação deles.
Dando sequência ao experimento, agora para a medição em corrente contínua, fez-se a retirada do driver de conversão das lâmpadas. A Figura 4 ilustra o driver de conversão e a lâmpada n. 1 após a retirada.

Após a retirada do driver de conversão efetuou-se a ligação positivo e negativo das lâmpadas, visto que sem o driver obtêm-se polaridade.

Para medir a eficiência das lâmpadas em corrente contínua, assim como na primeira etapa do experimento, colocou-se as lâmpadas sem o driver de conversão dentro da esfera integrada de Ulbricht. Os resultados inerentes às três lâmpadas, agora sem o driver de conversão, foram obtidos após aguardar o mesmo tempo necessário para estabilizar o fluxo de energia no experimento anterior (quinze minutos), quando foram realizadas as medições em corrente alternada.

Nesta etapa do experimento os valores de corrente, estabelecidos na fonte de CC da marca Chroma, modelo 62006-P-3008, foram os valores obtidos no início do experimento para cada lâmpada (Tabela 2).

Os resultados desses experimentos serão apresentados, avaliados e discutidos no tópico “Resultados e discussões”, posteriormente.

\section{Metodologia}

Para dimensionar um sistema fotovoltaico necessário para abastecer o sistema de iluminação em CC, considerou-se um ambiente residencial de $100 \mathrm{~m}^{2}$ iluminado somente com as lâmpadas do tipo n. 1 do experimento realizado em laboratório. 
Figura 3 - Esfera integrada de Ulbricht e a lâmpada n. 1 para medição de dados
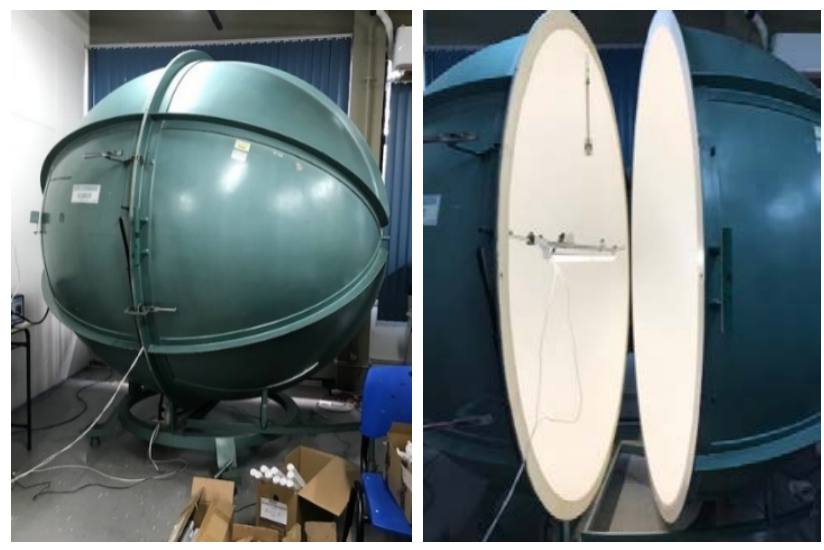

Figura 4 - Driver de conversão (à esquerda) e lâmpada n. 1 sem o driver (à direita)
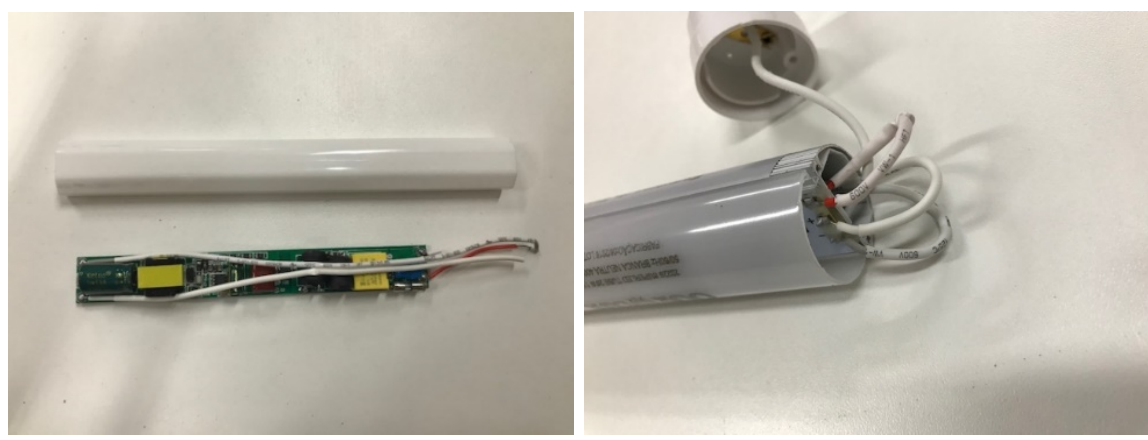

A avaliação da quantidade de lúmens e de lâmpadas necessárias ao ambiente residencial proposto neste experimento foi obtida de acordo com a CIE 89951 (ABNT, 2013).

A NBR que regia a iluminância em ambientes residenciais era a NBR 5413 (ABNT, 1992), a qual foi substituída pela NBR ISO 8995-1. Porém, a nova NBR trata apenas de iluminação em ambientes de trabalho, não abordando ambientes residenciais. Assim, para o presente artigo foi empregada a quantidade de lúmens necessária, de acordo com a nova NBR, a ambientes considerados equivalentes a uma residência. Por conseguinte, foi considerada a média entre a quantidade necessária a um saguão de entrada (100 lux) e sala de espera (200 lux), obtendo-se 150 lux (lúmens/m²) para um ambiente residencial.

Sendo assim, para uma residência de $100 \mathrm{~m}^{2}$, a quantidade de lúmens necessária, de acordo com a Equação 1, será de:

$L=150 \times A=150 \times 100=15.000$ lux. $m^{2}=$ $15.000 \mathrm{~lm}$

Eq. 1

Onde:

$\mathrm{L}$ = quantidade de lúmens necessária em um ambiente (lm); e

$\mathrm{A}$ = área da residência em metro quadrado $\left(\mathrm{m}^{2}\right)$.

A quantidade de lâmpada é calculada a partir da Equação 2:

$N$. de lâmpadas $=\frac{L}{L_{\text {lâmpada }}}=\frac{15.000}{1.767}=8,49=$

9 lâmpadas

Eq. 2

Onde:

L = quantidade de lúmens necessária em um ambiente (lm); e

Llâmpada = quantidade de lúmens que a lâmpada emite $(\mathrm{lm})$.

E a potência consumida pelo sistema é (Equação 3):

$P_{\text {sistema }}=P_{\text {lâmpada }} \times n^{\text {o }}$ de lâmpadas $=$ $15,7 \times 9=141,30 \mathrm{~W}$

Onde:

$\mathrm{P}_{\text {sistema }}=$ potência consumida pelo sistema em watts (W); e

$\mathrm{P}_{\text {lâmpada }}=$ potência da lâmpada medida em watts (W).

A Tabela 3 ilustra a quantidade de lúmens e de lâmpadas necessários ao ambiente residencial proposto, assim como a potência total do sistema. 
Tabela 3 - Dados do sistema de iluminação com a lâmpada n. 1 do experimento realizado em laboratório

\begin{tabular}{c|c|c|c|c|c}
\hline $\begin{array}{c}\text { Área da } \\
\text { residência } \\
\left(\mathbf{m}^{\mathbf{2}}\right)\end{array}$ & $\begin{array}{c}\text { Lúmens } \\
\text { necessários } \\
\mathbf{( l m})\end{array}$ & $\begin{array}{c}\text { Potência da } \\
\text { lâmpada (W) }\end{array}$ & $\begin{array}{c}\text { Lúmens da } \\
\text { lâmpada (lm) }\end{array}$ & $\begin{array}{c}\text { Quantidade de } \\
\text { lâmpadas } \\
\text { necessárias }\end{array}$ & $\begin{array}{c}\text { Potência total } \\
\text { do sistema } \\
\text { (W) }\end{array}$ \\
\hline 100 & 15.000 & 15,7 & 1.767 & 9 & 141,30 \\
\hline
\end{tabular}

Existem várias metodologias para se dimensionar um sistema fotovoltaico. A metodologia a ser seguida neste artigo é a do professor Marcelo Gradella Villalva, engenheiro eletricista, mestre e doutor em engenharia elétrica, professor da Faculdade de Engenharia Elétrica e de Computação (FEEC) da Unicamp. Marcelo Gradella Villalva é pesquisador nas áreas de conversão de energia elétrica e fontes renováveis e membro do IEEE (Institute of Electrical and Electronics Engineers).

Segundo Villalva (2015), para o dimensionamento de um sistema fotovoltaico é necessário primeiramente saber o consumo das cargas que devem ser alimentadas por ele. Para tal, torna-se necessário o conhecimento prévio dos equipamentos a serem abastecidos pelo sistema, assim como a média de horas que permanecerão ligados diariamente ou mensalmente.

Considerou-se, para o dimensionamento do sistema fotovoltaico, uma utilização média diária das lâmpadas do ambiente residencial igual a 5 (cinco) horas por dia e uma autonomia de 3 (três) dias para o sistema (caso o fornecimento de energia pelos painéis seja interrompido, ora por algum problema, ora por falta de irradiação causada por dias chuvosos ou nublados, o sistema continuará operando por três dias em condições normais).

A energia consumida diariamente por um aparelho nesse contexto por uma lâmpada é calculada de acordo com a expressão abaixo (Equação 4):

$E_{C}=P \times T=15,7 \times 5=78,5 \mathrm{Wh}$

Eq. 4

Onde:

$\mathrm{E}_{\mathrm{C}}=$ energia consumida em watts-hora (Wh);

$\mathrm{P}=$ potência em watts $(\mathrm{W})$; e

$\mathrm{T}=$ tempo de uso em horas durante um dia (h/dia).

A energia total consumida é obtida por meio da soma da energia individualmente consumida por todas as lâmpadas do sistema, logo (Equação 5):

$E_{T}=E_{C 1}+E_{C 2}+\cdots+E_{C n}=78,5 \times 9=$ 706,5 Wh

Eq. 5

Onde:

$\mathrm{E}_{\mathrm{T}}=$ energia total consumida pelo sistema em watts-hora (Wh); e
$\mathrm{E}_{\mathrm{C} 1}=\mathrm{E}_{\mathrm{C} 2}=\mathrm{E}_{\mathrm{Cn}}=$ energia consumida por cada lâmpada em watts-hora (Wh).

Após a determinação da carga total do sistema, é necessário dimensionar a bateria ou o banco de baterias. Alguns aspectos devem ser levados em consideração ao fazer o dimensionamento das baterias de um sistema fotovoltaico, como:

(a) quantificação de energia para o consumo diário das cargas;

(b) avaliação da autonomia do sistema, caso cesse a produção de energia pelos painéis em consequência de dias chuvosos ou nublados; e

(c) profundidade de descarga permitida para as baterias.

O primeiro aspecto citado acima é determinado por meio da energia total consumida pelo sistema, e definido segundo a Equação 5.

Em relação ao segundo aspecto é necessário conhecer previamente a forma de funcionamento do sistema fotovoltaico: o sistema deverá garantir integralmente a continuidade do serviço, não obstante a interrupção da geração de eletricidade; o sistema alimentará cargas, que na falta de produção de eletricidade devido a chuvas ou nuvens, os consumidores podem fazer o uso moderado dos equipamentos, fazendo com que não haja interrupção por completa da energia.

Esse aspecto deve ser bem avaliado, haja vista que, se as baterias forem superdimensionadas, o sistema pode encarecer muito e não ser utilizado de maneira coerente.

O último aspecto citado implica a durabilidade ou vida útil das baterias. Se as baterias tiverem um alto percentual de descarga diário, isso fará com que tenham o tempo de vida reduzido, acarretando a necessidade de troca em menor período de tempo. A alternativa de uma descarga diária percentualmente menor que acarrete, consequentemente, maior durabilidade implica a demanda de um maior número de baterias com o propósito de suprir a demanda da carga necessária. Assim sendo, o projetista deve avaliar o melhor custo-benefício em relação às alternativas colocadas.

A profundidade de descarga de uma bateria é indicada no manual. Como exemplo: uma bateria 
com profundidade de descarga de 50\% significa que, caso não haja produção de energia pelos painéis fotovoltaicos durante um período de dois dias, no final desse período a bateria estará descarregada em $50 \%$ e ela terá fornecido energia para as cargas durante esses dois dias.

Para achar a quantidade de baterias necessárias a um sistema, parte-se da energia total consumida pelo sistema multiplicada pela quantidade de dias durante o qual o sistema deverá funcionar sem a produção de energia feita pelos painéis, ou seja (Equação 6):

$E_{A}=E_{T} * n$. de dias de automação $=$ $706,5 \times 3=2119,5 \mathrm{Wh}$

Eq. 6

Onde:

$\mathrm{E}_{\mathrm{A}}=$ energia armazenada no banco de baterias em watts-hora (Wh); e

$\mathrm{E}_{\mathrm{T}}=$ energia total consumida pelo sistema em watts-hora (Wh).

A partir dessa metodologia foi possível obter os valores da energia total consumida (Equação 5), em Wh/dia, e da energia armazenada na bateria do sistema solar (Equação 6), tanto em Wh/dia quanto em $\mathrm{kWh} / \mathrm{mês.}$

A Tabela 4 ilustra os referidos valores.

As baterias em um sistema fotovoltaico podem ser ligadas em série e paralelo. As ligações em série permitem maiores tensões e em paralelo permitem maiores correntes.

No mercado as baterias são encontradas, em sua grande maioria, com tensões de $12 \mathrm{~V}, 24 \mathrm{~V}$ e $48 \mathrm{~V}$. Quando conectadas em série a tensão $(\mathrm{V})$ do banco de baterias é a soma da tensão de cada bateria e a corrente (A) é a mesma de uma só bateria, quando conectadas em paralelo a tensão é a mesma de uma só bateria e a corrente é a soma da corrente de cada bateria.

Para se determinar o número de baterias em série, faz-se (Equação 7):

$N_{B S}=V_{B A N C O} / V_{V B A T}=\frac{12}{12}=1$ unidade Eq. 7

Onde:

$\mathrm{N}_{\mathrm{BS}}=$ número de baterias ligadas em série;

$\mathrm{V}_{\text {BANCO }}=$ tensão do banco de baterias em volts (V); e

$\mathrm{V}_{\mathrm{VBAT}}=$ tensão da bateria utilizada em volts (V).
A tensão do banco de baterias deve levar em consideração a tensão das cargas a serem alimentadas. Caso essas voltagens sejam diferentes, é necessário utilizar conversores CC/CC para ajustar a tensão do sistema.

Posteriormente, determina-se a capacidade do banco de baterias como sendo (Equação 8):

$C_{B A N C O}=E_{A} / V_{B A N C O}==\frac{2119,5}{12}=176,63 \mathrm{Ah}$

Onde:

$\mathrm{C}_{\mathrm{B}}=$ capacidade do banco de baterias em ampèrehora (Ah);

$\mathrm{E}_{\mathrm{A}}=$ energia armazenada no banco de baterias em watts-hora (Wh); e

$\mathrm{V}_{\text {BANCO }}=$ tensão do banco de baterias em volts (V).

Para se determinar o número de baterias em paralelo, faz-se (Equação 9):

$$
\begin{aligned}
& N_{B P}= \\
& C_{B A N C O} / C_{B A T}==\frac{176,63}{115}=1,54(2 \text { conjunto })
\end{aligned}
$$

Onde:

$\mathrm{N}_{\mathrm{BP}}=$ número de conjuntos de baterias ligados em paralelo;

$\mathrm{C}_{\mathrm{BANCO}}=$ capacidade de carga do banco de baterias em ampère-hora (Ah); $\mathrm{e}$

$\mathrm{C}_{\mathrm{BAT}}=$ capacidade de carga de cada bateria em ampère-hora (Ah).

A quantidade de painéis fotovoltaicos irá depender de especificações técnicas que vêm previamente estipuladas pelos fabricantes, como a eficiência do módulo. O modelo de painel selecionado é o do fabricante Canadian, modelo CSI CS6P-255P, de material silício policristalino, cujas dimensões são 1.638 mm x 982 mm e eficiência de 15,85\%.

O município de Niterói está localizado a uma latitude de $22^{\circ} 53^{\prime} 00^{\prime \prime}$ sul e há uma longitude de 4306'13', oeste. Para que não haja falta de energia fornecida pelos painéis, é necessário que o sistema solar fotovoltaico seja dimensionado para o pior cenário de irradiação possível. De acordo com a Figura 5, a irradiação diária média da cidade no pior mês é de $3.320 \mathrm{~W} / \mathrm{m}^{2} /$ dia.

Tabela 4 - Dados do sistema de iluminação com a lâmpada 1 do experimento realizado em laboratório

\begin{tabular}{c|c|c|c|c}
\hline N. da lâmpada & $\begin{array}{c}\text { Potência do } \\
\text { sistema }(W)\end{array}$ & $E_{T}(W h / d i a)$ & $E_{a}(W h / d i a)$ & $E_{a}(\mathrm{kWh} / \mathbf{m e ̂ s})$ \\
\hline 1 & 133,28 & 706,5 & 2119,5 & 63,59 \\
\hline
\end{tabular}


Figura 5 - Irradiação diária média no município de Niterói

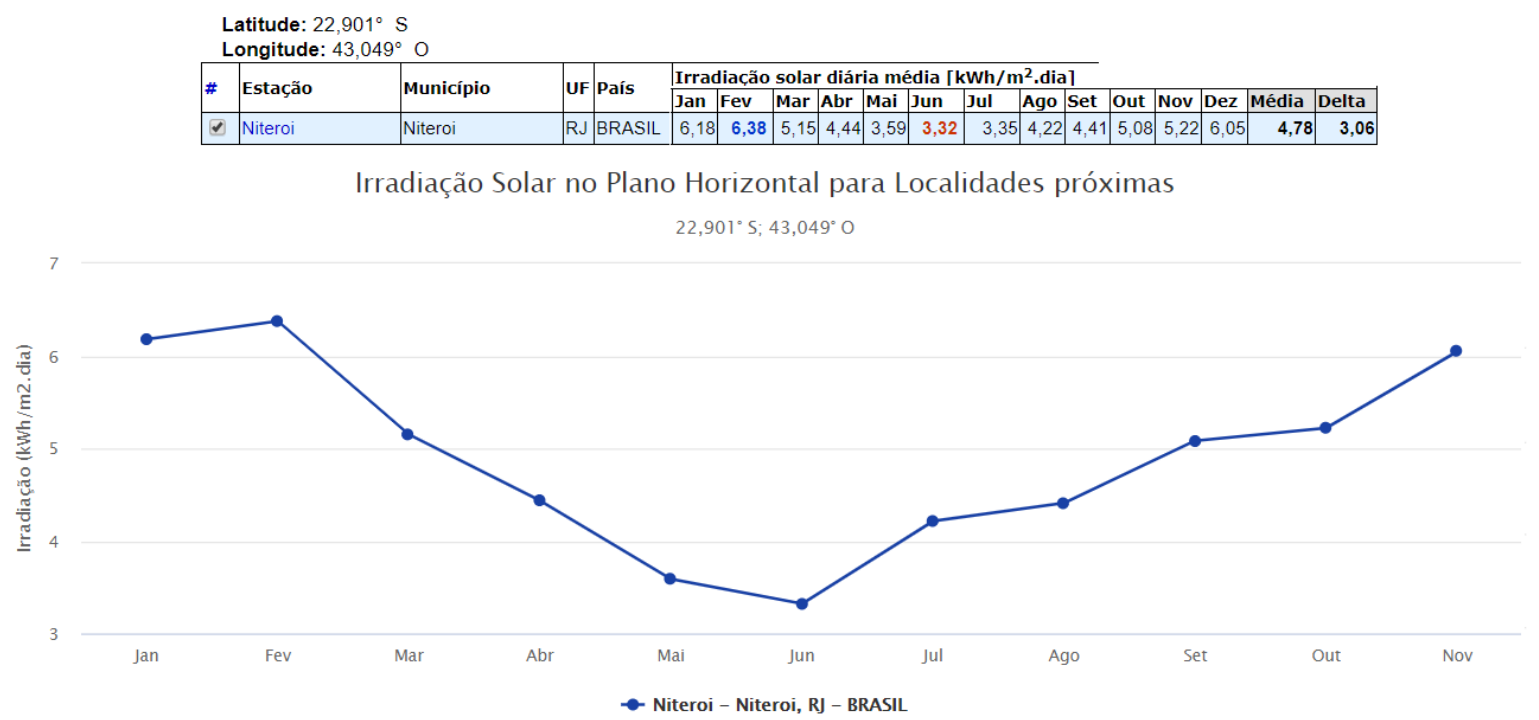

Fonte: CRESESB (2018).

Posto isso, o cálculo da energia produzida por um painel é feito por meio da Equação 10:

$E_{P}=E_{S} \times A_{M} \times \eta_{M}=$ $3.320 \times(1,638 \times 0,982) \times 15,85 \%=$ $846,43 W h$

Eq. 10

Onde:

$E_{\mathrm{P}}=$ energia produzida pelo painel diariamente em watts-hora (Wh);

$\mathrm{E}_{\mathrm{S}}=$ irradiação diária em watts-hora por metro quadrado por dia (Wh/m²/dia);

$\mathrm{A}_{\mathrm{M}}$ = área da superfície do módulo em metro quadrado $\left(\mathrm{m}^{2}\right)$; e

$\eta$ м $=$ eficiência do módulo (\%).

Deste modo, o número de painéis necessários para o sistema será (Equação 11):

$N=E_{T} / E_{P}==\frac{706,5}{846,43}=0,83$ (1 unidade) Eq. 11

Onde:

$\mathrm{N}$ = número de painéis empregados no sistema;

$\mathrm{E}_{\mathrm{T}}=$ energia total consumida pelo sistema em watts-hora (Wh); e

$\mathrm{E}_{\mathrm{P}}=$ energia produzida pelo painel diariamente em watts-hora (Wh).

O controlador de carga de um sistema fotovoltaico deve ser selecionado levando-se em conta a tensão e a corrente máxima fornecida pelos módulos fotovoltaicos. A corrente máxima fornecida pelo módulo fotovoltaico é de 8,43 A.

A corrente máxima fornecida deve ser corrigida com um fator de segurança, para garantir a funcionalidade do controlador de carga, fazendo com que a corrente máxima não exceda em nenhuma hipótese. O fator de segurança é um número prático especificado de acordo com o projetista. É utilizado neste projeto uma correção de 30\%, passando, desse modo, a corrente máxima de projeto a ser de 10,96 A.

O sistema fotovoltaico necessário para suprir a necessidade dessa energia calculada anteriormente (Tabela 4) deve ser composto por:

(a) 1 módulo fotovoltaico de $255 \mathrm{Wp}$;

(b) 2 baterias de $12 \mathrm{~V}$ com capacidade de carga de $115 \mathrm{Ah}$;

(c) 1 conversor CC/CC; e

(d) 1 controlador de carga $12 \mathrm{~V} / 24 \mathrm{~V}$.

Com a finalidade de obter-se o preço total de um sistema fotovoltaico modelo, para posterior cálculo do tempo de retorno do investimento, fez-se a escolha dos itens do sistema fotovoltaico de acordo com os fabricantes abaixo:

(a) 1 módulo fotovoltaico do fabricante Canadian, modelo CSI CS6P-255P;

(b) 2 baterias fabricante Freedom, modelo DF2000;

(c) 1 conversor CC/CC fabricante Boost, modelo Step-up; e

(d) 1 controlador de carga fabricante Epsolar Landstar, modelo LS2024B 20A 12 V/24 V.

A Tabela 5 mostra o custo do sistema solar fotovoltaico acima especificado. Esse custo foi obtido a partir de pesquisa de valor de mercado dos itens que o compõem. 


\section{Resultados e discussões}

A Tabela 6 mostra os resultados dos parâmetros elétricos obtidos com o experimento nas lâmpadas n. $1 ;$ n. 2 e n. 3 realizados no laboratório de luminotécnica.

Observa-se, na Tabela 6, que a potência efetiva, ou seja, a potência medida da lâmpada, diminui em todos os casos após a retirada dos drivers de conversão, concluindo que eles, conforme esperado, consomem uma parte da energia disponibilizada pelas lâmpadas. A saber, as reduções nas lâmpadas foram de 19,45\%; 8,24\% e $10,07 \%$ nas lâmpadas n. 1, n. 2 e n. 3, respectivamente.

A Tabela 7 abaixo mostra os resultados de fluxo luminoso e eficácia luminosa (parâmetros radiométricos e fotométricos) das lâmpadas dos experimentos realizados.

A partir da Tabela 7 , é possível perceber que a eficácia de todas as lâmpadas aumenta quando o driver é retirado, passando ela a operar em CC e, consequentemente, reduzindo as perdas devido à utilização dos drivers. Isso se deve ao fato da potência efetiva diminuir com a retirada do driver (Tabela 3), visto que a eficácia luminosa da lâmpada é dada por (Equação 12):
Eficácia luminosa $=\frac{\text { Fluxo luminoso }}{\text { Potência } \text { efetiva }}(\mathrm{lm} / \mathrm{W})$ Eq. 12

Observa-se, ainda na Tabela 7, que as lâmpadas 2 e 3 , cujas potências nominais especificadas pelo fabricante são iguais, têm acréscimos percentuais de suas eficácias muito próximos (10,24\% e 11,63\%, respectivamente), em consequência da retirada dos seus drivers de transformação. Para a lâmpada 1, esse acréscimo torna-se ainda mais significativo (32,02\%).

A Tabela 8 mostra os resultados dos parâmetros colorimétricos, IRC e CCT, obtidos do experimento com as lâmpadas. O IRC é a medida de correspondência entre a cor real de um objeto ou superfície e sua aparência diante de uma fonte luminosa, enquanto o CCT expressa a aparência da cor emitida pela fonte de luz.

Verifica-se, na Tabela 8, que, após da retirada dos drivers, tanto no índice de reprodução de cor quanto na temperatura de cor não foram obtidas variações significativas (menos de 1\%). Pode-se, dessa maneira, concluir que sem os drivers as lâmpadas mantêm suas características relativas à cor, não alterando características importantes para utilização em projetos luminotécnicos.

Tabela 5 - Custo do sistema solar fotovoltaico

\begin{tabular}{c|c|c|c}
\hline Item & Quantidade (unid.) & Preço de mercado (R\$) & Preço total (R\$) \\
\hline Módulo fotovoltaico & 1 & 756,06 & 756,06 \\
Bateria & 2 & 680,34 & $1.360,68$ \\
Conversor CC/CC & 1 & 152,44 & 152,44 \\
Controlador de carga & 1 & 114,63 & 114,63 \\
\hline \multicolumn{2}{l}{} & Total & $\mathbf{2 . 3 8 3 , 8 1}$ \\
\hline
\end{tabular}

Tabela 6 - Parâmetros elétricos das lâmpadas n. 1, n. 2 e n. 3

\begin{tabular}{c|c|c|c|c|c|c}
\hline \multirow{2}{*}{$\begin{array}{c}\text { N. da } \\
\text { lâmpada }\end{array}$} & \multirow{2}{*}{$\begin{array}{c}\text { Potência } \\
\text { nominal } \\
\text { (W) }\end{array}$} & \multicolumn{2}{|c|}{ CA } & \multicolumn{2}{|c}{ CC } & Redução da \\
efetiva (W) & $\begin{array}{c}\text { Corrente } \\
\text { (mA) }\end{array}$ & $\begin{array}{c}\text { Potência } \\
\text { efetiva (W) }\end{array}$ & $\begin{array}{c}\text { Corrente } \\
\text { (mA) }\end{array}$ & $\begin{array}{c}\text { potência } \\
\text { (CA-CC) \% }\end{array}$ \\
\hline 1 & 20,0 & $\mathbf{1 9 , 5 4}$ & 159,5 & $\mathbf{1 5 , 7 4}$ & 217,7 & $\mathbf{1 9 , 4 5}$ \\
\hline 2 & 10,0 & $\mathbf{9 , 7 1}$ & $\begin{array}{c}77,4 \\
73\end{array}$ & $\mathbf{8 , 9 1}$ & 121,4 & $\mathbf{8 , 2 4}$ \\
\hline 3 & 10,0 & $\mathbf{9 , 1 4}$ & 73,5 & $\mathbf{8 , 2 2}$ & 144,3 & $\mathbf{1 0 , 0 7}$ \\
\hline
\end{tabular}

Tabela 7 - Parâmetros radiométricos e fotométricos das lâmpadas n. 1, n. 2 e n. 3.

\begin{tabular}{c|c|c|c|c|c}
\hline \multirow{2}{*}{$\begin{array}{c}\text { N. da } \\
\text { lâmpada }\end{array}$} & \multicolumn{2}{|c|}{ CA } & \multicolumn{2}{c|}{ CC } & Aumento da \\
\cline { 2 - 5 } & $\begin{array}{c}\text { Fluxo luminoso } \\
\mathbf{( l m})\end{array}$ & $\begin{array}{c}\text { Eficácia } \\
(\mathbf{l m} / \mathbf{W})\end{array}$ & $\begin{array}{c}\text { Fluxo } \\
\text { luminoso (lm) }\end{array}$ & $\begin{array}{c}\text { Eficácia } \\
(\mathbf{l m} / \mathbf{W})\end{array}$ & $\begin{array}{c}\text { efia } \\
\text { (CA-CC) \% }\end{array}$ \\
\hline 1 & $1.661,4$ & $\mathbf{8 5 , 0 3}$ & $1.767,0$ & $\mathbf{1 1 2 , 2 6}$ & 32,02 \\
2 & 915,5 & $\mathbf{9 4 , 2 9}$ & 926,2 & $\mathbf{1 0 3 , 9 5}$ & $\mathbf{1 0 , 2 4}$ \\
3 & $1.080,1$ & $\mathbf{1 1 8 , 1 8}$ & $1.084,5$ & $\mathbf{1 3 1 , 9 3}$ & $\mathbf{1 1 , 6 3}$ \\
\hline
\end{tabular}


Tabela 8 - Parâmetros colorimétricos das lâmpadas n. 1, n. 2 e n. 3.

\begin{tabular}{c|c|c|c|c}
\hline \multirow{2}{*}{$\begin{array}{c}\text { N. da } \\
\text { lâmpada }\end{array}$} & \multicolumn{2}{|c|}{ CA } & \multicolumn{2}{c}{ CC } \\
\cline { 2 - 5 } & IRC & CCT (K) & IRC & CCT (K) \\
\hline 1 & 83,8 & 4010 & 83,5 & 4039 \\
2 & 83,5 & 4073 & 83,6 & 4082 \\
3 & 86,1 & 6581 & 86,2 & 6567 \\
\hline
\end{tabular}

Para analisar a viabilidade do sistema solar proposto é preciso considerar algumas variáveis como, por exemplo:

(a) o preço da tarifa de energia elétrica cobrada na região onde o sistema solar for instalado. Quanto maior o valor dessa tarifa, mais viável financeiramente é a instalação de energia solar, pois a energia que você produz se traduz em uma economia financeira maior;

(b) a variável da alta de energia; ou seja, a inflação energética (variação do preço da tarifa de energia no tempo);

(c) As variáveis técnicas, como o tamanho do sistema solar (quanto maior um sistema fotovoltaico, menor é o seu custo por watt) e a solarimetria da região (a quantidade de radiação interfere diretamente na geração de energia ao longo do ano, o que gera uma variação no retorno do investimento em energia solar); e

(d) a variável do ganho patrimonial; isto é, o imóvel que possui um sistema solar fotovoltaico é avaliado com um valor maior comparado a um imóvel de iguais características.

Para a avaliação da viabilidade do sistema proposto neste artigo será considerada a variável da alta de energia, a inflação energética. A variável do preço da tarifa foi determinada a partir do momento que se especificou o local de instalação do sistema proposto, e as variáveis técnicas também já foram definidas durante o dimensionamento. Já a variável do ganho patrimonial, por possuir variáveis imensuráveis, não será considerada no cálculo, apesar de contribuir sempre para um tempo de retorno de investimento menor.

Os métodos mais utilizados, com base no fluxo de caixa dos projetos, são: período de payback simples, período de payback descontado, taxa interna de retorno (TIR) e valor presente líquido (VPL).

No estudo do presente artigo será utilizado o payback simples, a TIR e o VPL para análise de investimento do projeto em questão, levando em consideração uma taxa mínima de atratividade (TMA) que será baseada nos ganhos de uma aplicação financeira. Como o estudo está baseado em uma unidade residencial, será adotada uma taxa que esteja dentro desse perfil de consumidor, e que atenda aos requisitos mínimos; ou seja, deverá ser uma taxa de juros equivalente à rentabilidade das aplicações correntes, seguras e de baixo risco. Assim, será utilizada a taxa de 6,60\% (INSTITUTO..., 2018) correspondente a uma aplicação de caderneta de poupança no ano de 2017 (ano anterior à instalação do sistema proposto).

O custo do investimento foi de R\$2.383,81 (Tabela 5). A duração de um sistema solar é considerada, pelos fabricantes, de 25 anos, que é o tempo de garantia das placas. Assim, será considerado um custo de manutenção do sistema de $2 \%$ ao ano, gerando um investimento de R\$ 3.575,72.

O valor da energia cobrada no município escolhido para a instalação do sistema solar variou, entre janeiro de 2013 a dezembro de 2017, de acordo com os seguintes valores, segundo mostra a Tabela 9.

Para fazer a análise do payback do projeto é necessário aplicar uma tabela demonstrando a depreciação do valor investido $\mathrm{x}$ valor economizado na conta de luz da residência. Para melhor entendimento, será apresentado o fluxo de caixa do período em que o projeto estará em atividade.

O fluxo de caixa de cada mês será o valor da média da tarifa dos últimos cinco anos (janeiro/2013 até dezembro/2017), sendo esse o valor de $\mathrm{R} \$ 0,6786$, multiplicado pela quantidade de kWh que será necessário para a residência por mês para abastecer o sistema de iluminação, sendo 63,59 kWh/mês, obtendo-se o valor de R\$43,15. Para o fluxo de caixa de cada ano, apenas multiplica-se esse valor por 12, encontrando $\mathrm{R} \$ 517,83$. O sistema de payback é mostrado na Tabela 10.

Conforme dados da Tabela 10, verifica-se que o payback do projeto de instalação é dado no ano 6, ou seja, o projeto terá o retorno do investimento no sexto ano após sua instalação. Após esse período, conta-se que os demais valores dos próximos fluxos de caixa serão de lucro para o investidor; ou seja, no final dos 25 anos, a implantação do sistema renderá $\mathrm{R} \$ 9.370,03$. 
Tabela 9 - Preço (R\$) do kWh/mês de 2013 até 2017, de acordo com a concessionária Enel, distribuidora da região

\begin{tabular}{c|c|c|c|c|c}
\hline Mês/Ano & $\mathbf{2 0 1 3}$ & $\mathbf{2 0 1 4}$ & $\mathbf{2 0 1 5}$ & $\mathbf{2 0 1 6}$ & $\mathbf{2 0 1 7}$ \\
\hline Janeiro & 0,6783 & 0,6101 & 0,5816 & 0,8609 & 0,8512 \\
Fevereiro & 0,5373 & 0,5728 & 0,6327 & 0,8331 & 0,8734 \\
Março & 0,5057 & 0,5797 & 0,8114 & 0,8020 & 0,8733 \\
Abril & 0,5160 & 0,5687 & 0,8551 & 0,8333 & 0,7423 \\
Maio & 0,5369 & 0,5687 & 0,7733 & 0,7425 & 0,7121 \\
Junho & 0,5276 & 0,5213 & 0,7410 & 0,7005 & 0,6922 \\
Julho & 0,5188 & 0,5483 & 0,7391 & 0,6722 & 0,6876 \\
Agosto & 0,5354 & 0,4748 & 0,7395 & 0,7136 & 0,7087 \\
Setembro & 0,5315 & 0,5000 & 0,7262 & 0,7160 & 0,7105 \\
Outubro & 0,5701 & 0,6764 & 0,8575 & 0,7195 & 0,7147 \\
Novembro & 0,5040 & 0,6065 & 0,8498 & 0,7450 & 0,7351 \\
Dezembro & 0,6260 & 0,5513 & 0,8271 & 0,8548 & 0,7229 \\
\hline
\end{tabular}

Tabela 10 - Fluxo de caixa e payback (R\$)

\begin{tabular}{c|c|c|c}
\hline & Valor investido no projeto & Fluxo de caixa & Payback \\
\hline Ano 0 & $-3.575,72$ & & \\
Ano 1 & 517,83 & $-3.057,89$ \\
Ano 2 & 517,83 & $-2.540,06$ \\
Ano 3 & 517,83 & $-2.022,23$ \\
Ano 4 & 517,83 & $-1.504,40$ \\
Ano 5 & 517,83 & $-986,57$ \\
Ano 6 & 517,83 & $-468,74$ \\
Ano 7 & 517,83 & 49,09 \\
Ano 8 & 517,83 & 566,92 \\
Ano 9 & 517,83 & $1.084,75$ \\
Ano 10 & 517,83 & $1.602,58$ \\
Ano 11 & 517,83 & $2.120,41$ \\
Ano 12 & 517,83 & $2.638,24$ \\
Ano 13 & 517,83 & $3.156,07$ \\
Ano 14 & 517,83 & $3.673,90$ \\
Ano 15 & 517,83 & $4.191,73$ \\
Ano 16 & 517,83 & $4.709,56$ \\
Ano 17 & 517,83 & $5.227,39$ \\
Ano 18 & & 517,83 & $5.745,22$ \\
Ano 19 & & 517,83 & $6.263,05$ \\
Ano 20 & & 517,83 & $6.780,88$ \\
Ano 21 & 517,83 & $7.298,71$ \\
Ano 22 & & 517,83 & $7.816,54$ \\
Ano 23 & 517,83 & $8.334,37$ \\
Ano 24 & 517,83 & $8.852,20$ \\
Ano 25 & 517,83 & $9.370,03$ \\
\hline
\end{tabular}

Porém, somente a análise do payback simples não fornece condições para a aceitação do projeto por não levar em consideração o valor do dinheiro no decorrer do projeto; ou seja, o valor do dinheiro no tempo. Desse modo, o próximo passo é realizar a análise do investimento considerando o valor do dinheiro durante a aplicação do projeto, que é de 25 anos.

Para isso, o cálculo do VPL e da TIR dará condições de se tomar a decisão em aceitar ou rejeitar o projeto. O VPL é encontrado a partir da seguinte Equação 13 (ASSAF NETO; LIMA, 2014):

$V P L=-C F_{o}+\sum \frac{C F j}{(1+i)^{n}}$ Eq. 13

Onde:

$\mathrm{CF}_{\mathrm{o}}=$ Fluxo de caixa inicial;

$\mathrm{CF}_{\mathrm{j}}=$ Fluxos de caixa esperado; 
i = Taxa de desconto ou TMA (taxa mínima de atratividade); e

$\mathrm{n}$ = Período de tempo.

Utilizando-se a TMA como 6,60\% e o período de tempo de 25 nos, o valor do VPL encontrado é de $\mathrm{R} \$ 2.682,69$. Para que o projeto seja aceito, o VPL deverá ter o valor positivo, ou seja, VPL $>0$.

Seguindo com a análise, a fórmula para se obter o valor da TIR é representa pela Equação 14 (ASSAF NETO; LIMA, 2014):

$\sum \frac{C F_{n}}{(1+T I R)^{n}}-C F_{o}=0$

Onde:

TIR = Taxa interna de retorno;

$\mathrm{CF}_{\mathrm{o}}=$ Fluxo de caixa inicial;

$\mathrm{CF}_{\mathrm{n}}=$ Fluxo de caixa em cada período; $\mathrm{e}$

$\mathrm{n}$ = Período do movimento no fluxo de caixa.

Utilizando-se os valores já especificados, o valor da TIR é de 13,93\%, que, consequentemente, é maior do que a taxa de atratividade do investimento estabelecida de 6,60\%.

Portanto, analisando-se os valores encontrados de payback, VPL e TIR é possível concluir que a implantação do sistema fotovoltaico é vantajosa para as condições em questão.

\section{Conclusão}

Os valores obtidos no experimento realçam as vantagens da utilização das lâmpadas de LED em corrente contínua e sem drivers de conversão, uma vez que as perdas nas conversões em consequência do uso demonstraram-se significativas. É possível observar na Tabela 6 e 7 que as lâmpadas sem os drivers possuem uma potência menor, utilizando menor quantidade de energia, sendo, consequentemente, mais eficientes.

Na Alemanha, a região mais ensolarada recebe um índice de radiação solar 40\% menor que o índice da região menos ensolarada do Brasil (CABRAL; TORRES; SENNA, 2013); e, segundo Boeke, Wendt e Ysebood (2013), na Alemanha, um protótipo de um sistema de iluminação com lâmpadas de LED em CC utilizando energia fotovoltaica gerou uma economia de $15 \%$. Comparando com os dados obtidos no experimento desse artigo é possível concluir que o Brasil tem grande potencial para avançar nessa área.

Além das vantagens em relação à eficiência das lâmpadas de LED, quando é feita a retirada do driver, um outro aspecto que contribui para realçar a importância dos resultados deste experimento é que, em escala industrial, para a fabricação de lâmpadas de LED em grande quantidade, o preço das lâmpadas tende a reduzir, já que, por dispensarem os drivers, apresentam maior simplicidade no seu processo de montagem. Isso viabilizará, assim, maior uso de lâmpadas de LED mais eficientes e a um menor custo para a iluminação.

Considerando-se a utilização de LED em corrente contínua e, com o consequente incremento no uso de circuitos em corrente contínua, as fontes renováveis de energia, tais como a fotovoltaica, poderão ser mais facilmente incorporadas.

Pelo fato de não haver a necessidade dos processos de conversão, a presença do inversor é dispensada, o que contribui, assim, para redução nas perdas causadas pelos sistemas atualmente em uso e também no custo do sistema fotovoltaico em si. O uso de inversores no sistema fotovoltaico dimensionado no experimento disposto nesse artigo pode variar entre $\mathrm{R} \$ 1.500,00$ e $\mathrm{R} \$ 3.000,00$; ou seja, seu peso em relação ao sistema varia de $39 \%$ e 56\%, de acordo com a Portal Solar Ltda. (2019).

As novas tecnologias, em sentido contrário ao sentido da inflação e da crise energética brasileira e mundial, diminuem os custos e investimentos dos sistemas solares fotovoltaicos. Conforme mostrado em Boeke, Wendt E Ysebood (2013), maior redução nos custos dos investimentos de sistemas solares vai motivar novas tecnologias e aplicações do autoconsumo de energia solar. Esse fator é decisivo na aquisição desses tipos de geradores, pois aceleram e encurtam o tempo de payback. ,0

Não obstante os pontos favoráveis à implantação de sistemas distribuídos em corrente contínua, é importante ressaltar que a sua viabilidade, a priori, encontra-se comprometida em função da necessidade da criação de um mercado de aparelhos eletrônicos que se adéquem a essa modalidade de corrente. Nesse trabalho são as luminárias tipo LED que deverão passar por modificações para se adequar a esse novo sistema.

Essas modificações podem ser resumidas na retirada dos inversores que transformam a tensão CA da rede em tensão CC para que tal aparelho atenda a suas funcionalidades. De maneira semelhante, é necessário um mercado de equipamentos voltados para a utilização exclusiva em circuitos de corrente contínua.

Apesar dos bons resultados verificados aparentemente, não se pode generalizar a aplicação de LEDs sem drivers como a melhor alternativa sempre. Em casos particulares de sistemas de menor porte, sobretudo sistemas isolados alimentados em corrente contínua, como é o caso de sistemas isolados alimentados por painéis fotovoltaicos, os 
LEDs sem drivers poderiam ser uma alternativa interessante.

Todavia, em sistemas maiores ou conectados à rede elétrica, os drivers mostram-se necessários, não apenas como conversores de corrente CA em CC, mas também para melhoria da qualidade de energia, o que inclui parâmetros como fator de potência adequado, baixa distorção harmônica, entre outros. Além disso, os drivers podem agregar funções atrativas, como facilidade de dimerização e controle à distância (telegerenciamento). Essas funcionalidades podem ser particularmente desejáveis em sistemas de iluminação pública com LEDs.

Além disso, deve-se criar normas que guiem a construção segura desses sistemas, bem como mão de obra qualificada para execução e implementação dos projetos.

\section{Referências}

ALVES, J. E. D. A Demanda de Energia e o Crescimento das Fontes Renováveis Até 2035. Eco Debate. Disponível em:

$<$ https://www.ecodebate.com.br/2017/02/24/dema nda-de-energia-e-o-crescimento-das-fontesrenovaveis-ate-2035-artigo-de-jose-eustaquiodiniz-alves/>. Acesso em: 10 jul. 2018.

ASSAF NETO, A.; LIMA, F. G. Curso de Administração Financeira. 3. ed. São Paulo: Atlas, 2014.

ASSOCIAÇÃO BRASILEIRA DE NORMAS TÉCNICAS. NBR 5413: iluminância de interiores. Rio de Janeiro, 1992.

\section{ASSOCIAÇÃO BRASILEIRA DE NORMAS}

TÉCNICAS. NBR 8995-1: iluminação em ambientes de trabalho. Rio de Janeiro, 2013.

BOEKE, U.; WENDT, M.; YSEBOOD, L. DC Power Supply of Systems of LEDs with Network Power and of Solar Panels. In: EUROPEAN CONFERENCE ON POWER ELETRONICS AND APPLICATIONS, 14., London, 2013. Proceedings... London, 2013.

CABRAL, I. S.; TORRES, A. C.; SENNA, P. R. Energia Solar: análise comparativa entre Brasil e Alemanha. In: CONGRESSO BRASILEIRO DE GESTÃO AMBIENTAL, Salvador, 2013. Anais... 2013.

EMPRESA DE PESQUISA ENERGÉTICA. Plano Decenal de Expansão da Energia 2024. Ministério de Minas e Energia, Brasília, 2015.
GAVIRIA, L. R.; PEREIRA, F. O. R.; MIZGIER, M. O. Influência da Configuração Urbana na Geração Fotovoltaica com Sistemas Integrados às Fachadas. Ambiente Construído, Porto Alegre, v. 13, n. 4, p. 7-23, out./dez. 2013.

IMHOFF, J. Desenvolvimento de Conversores Estáticos para Sistemas Fotovoltaicos Autônomos. Santa Maria, 2007. Dissertação (Mestrado em Engenharia Civil) - Escola de Engenharia, Universidade Federal de Santa Maria, Santa Maria, 2007.

\section{INSTITUTO PARANAENSE DE}

DESENVOLVIMENTO ECONÔMICO E SOCIAL. Rendimentos da Caderneta de Poupança - Brasil - Janeiro 2007 - Dezembro 2018. Disponível em:

$<$ http://www.ipardes.gov.br/pdf/indices/poupanca. pdf $>$. Acesso em: 23 jan. 2019.

LENOIR, A.; THELLIER, F.; GARDE, F. Towards Net Zero Energy buildings in hot climate: part 2: experimental Feedback. ASHRAE 2011. Disponível em: <http://task40.ieashc.org/data/sites/1/publications/DC-TP7-Garde2011-11.pdf>. Acesso em: 15 jan. 2019.

\section{MASARO, L. Um Outro Fim do Mundo é}

Possível: energia, entropia e o colapso da civilização industrial. São Paulo, 2016. Tese (Doutorado em Filosofia) - Faculdade de Filosofia, Letras e Ciências Humanas, São Paulo, 2016.

MONTEIRO, R. V. A.; CARVALHO, B. C.; NOGUEIRA, F. Drivers de Lâmpadas de LED : topologias, aplicações e desempenho. Engineering and Science, v. 2, p. 1-7, nov. 2014.

MOREIRA, H. M.; GIOMETTI, A. L. B. R. Protocolo de Quioto e as Possibilidades de Inserção do Brasil no Mecanismo de Desenvolvimento Limpo por Meio de Projetos em Energia Limpa: contexto internacional. Instituto de Relações Internacionais, v. 30, n. 1, p. 9-47, 2008.

MOURA, M. Iluminação: análise de cenários utilizando a tecnologia LED. Niterói, 2015. 208 f. Tese (Doutorado em Engenharia Civil) Universidade Federal Fluminense, Niterói, 2015.

NOGUEIRA, F. J. Influência da Temperatura no Desempenho de Luminárias LED. In: INTERNATIONAL CONFERENCE ON INDUSTRY APPLICATIONS, Fortaleza, 2012. Proceedings... Fortaleza, 2012. 
OLIVEIRA, T. R. de. Distribuição Local de Energia em Corrente Contínua: estudo, desenvolvimento e implementação de um protótipo de nanorrede C.C. dedicado aos setores residencial e comercial. Belo horizonte, 2016. $336 \mathrm{f}$. Tese (Doutorado em Engenharia Civil) - Escola de Engenharia, Universidade Federal de Minas Gerais, Belo Horizonte, 2016.

PINTO, R. A. Projeto e Implementação de Lâmpadas para Iluminação de Interiores Empregando Diodos Emissores de Luz (LEDS). Santa Maria, 2008. 138 f. Dissertação (Mestrado em Engenharia Civil) - Escola de Engenharia, Universidade Federal de Santa Maria, Santa Maria, 2008.
PORTAL SOLAR LTDA. O Inversor Solar. Disponível em:

<https://www.portalsolar.com.br/o-inversorsolar.html>. Acesso em: 15 jan. 2019.

\section{U.S. ENERGY INFORMATION AND}

ADMINISTRATION. International Energy

Outlook 2017. Sep. 2017. Disponível em:

$<$ https://www.eia.gov/outlooks/ieo/pdf/0484(2017) .pdf>. Acesso em: 10 jul. 2018.

VILLALVA, M. G. Energia Solar Fotovoltaica: conceitos e aplicações / sistemas isolados e conectados à rede. 2. ed. São Paulo: Érica, 2015.

\section{Carolina Lannes Boquimpani}

Pós-graduação em Engenharia Civil, Centro Tecnológico | Universidade Federal Fluminense | Rua Passos da Pátria 156, Bloco D, São Domingos | Niteroi - RJ - Brasil| CEP 24210-020 | Tel.: (21) 2629-5410| E-mail: carolinaboquimpani@gmail.com

\section{Ana Lúcia Torres Seroa da Motta}

Escola de Arquitetura e Urbanismo, Centro Tecnológico | Universidade Federal Fluminense | Tel.: (21) 2629-5402 | E-mail: anaseroa@hotmail.com

\section{Osvaldo Luiz de Carvalho Souza}

Escola de Arquitetura e Urbanismo, Centro Tecnológico | Universidade Federal Fluminense | Tel.: (21) 2629-5746 | E-mail: osvaldoluiz246@gmail.com

\section{Carlos Henriques Ventura do Rosário Oliveira}

Centro Tecnológico | Universidade Federal Fluminense | Tel.: (21) 2620-7070| E-mail: carloshneriques@id.uff.br

\section{Revista Ambiente Construído}

Associação Nacional de Tecnologia do Ambiente Construído

Av. Osvaldo Aranha, 99 - 3o andar, Centro

Porto Alegre - RS - Brasil

$$
\text { CEP } 90035-190
$$

Telefone: +55 (51) 3308-4084

Fax: +55 (51) 3308-4054

www. seer. ufrgs. br/ ambienteconstruido

E-mail: ambienteconstruido@ufrgs.br 